\title{
Humoral response to viral vector COVID-19 vaccine in hemodialysis patients
}

\author{
Niranjan Raja, Arul Rajagopalan, Jegan Arunachalam, Arun Prasath, Rakesh Durai, Manorajan Rajendran \\ Department of Nephrology, Madurai Medical College, Madurai, India
}

Background: The coronavirus disease 2019 (COVID-19) vaccine is not readily available in many countries where dosing interval is spaced more than ideal. Patients with chronic kidney disease, especially those on maintenance hemodialysis, have a tendency for a reduced immune response. This study was undertaken to demonstrate the distinct humoral immune response to the viral vector COVID-19 vaccine in patients with kidney failure receiving maintenance hemodialysis.

Methods: The study was carried out with two cohorts: 1) patients receiving maintenance hemodialysis and 2) healthcare workers from the same dialysis center as controls, each group with 72 subjects. Participants received a dose of Covishield ChAdOx $1 \mathrm{nCoV}-19$ coronavirus vaccine. The humoral immunological response was determined using electrochemiluminescence immunoassay which quantitatively measures antibodies to the severe acute respiratory syndrome coronavirus 2 spike protein receptor-binding domain.

Results: All study subjects in the control group developed a humoral response (antibody titer of $\geq 0.8 \mathrm{U} / \mathrm{mL}$ ), while only 64 of 72 in the dialysis group (88.9\%) were responders. Age $(\rho=-0.234, p=0.04)$ and sodium level $(\rho=0.237, p=0.04)$ correlated with low antibody titer in bivariate analysis. In multivariate analysis, only age (odds ratio, 1.10; 95\% confidence interval, 1.01-1.22; $p=0.045$ ) was associated with nonresponders.

Conclusion: Our study demonstrated a weak antibody response of hemodialysis patients to the viral vector COVID-19 vaccine. Older age was associated with nonresponders. Evaluation of both humoral and cellular immunity after the second vaccine dose and serial antibody titers can help determine the need for booster shots.

Keywords: Antibody formation, Secondary immunization, Chronic kidney failure, COVID-19, Hemodialysis

\section{Introduction}

The coronavirus disease 2019 (COVID-19) pandemic has severely affected countries worldwide. Vaccination of the majority of the population is an effective method to end the pandemic. However, vaccine availability remains unfeasible in many parts of the world, especially in developing nations. Although in many countries, including India, the replication-defective viral vector vaccine, ChAdOxl $\mathrm{nCoV}$ 19 (Oxford-AstraZeneca), was administered initially as two doses 4 weeks apart, it was later spaced to 12 to 16 weeks for better coverage of the population with at least a single dose and due to improved efficacy with the increased interval between doses [1]. The delay of the second dose

Received: August 19, 2021; Revised: October 27, 2021; Accepted: October 31, 2021

Correspondence: Niranjan Raja

Department of Nephrology, Madurai Medical College, Madurai Medical College, Panagal road, Alwarpuram, Madurai, Tamil Nadu 625020, India

E-mail: niranjan_raja@hotmail.com

ORCID: https://orcid.org/0000-0001-6866-4098

Copyright (@ 2022 by The Korean Society of Nephrology

(a) This is an Open Access article distributed under the terms of the Creative Commons Attribution Non-Commercial and No Derivatives License (http:// creativecommons.org/licenses/by-nc-nd/4.0/) which permits unrestricted non-commercial use, distribution of the material without any modifications, and reproduction in any medium, provided the original works properly cited. 
might be appropriate for the general population; however, patients with chronic kidney disease (CKD), especially those receiving maintenance hemodialysis, tend to have a reduced immune response, as evidenced with the hepatitis $B$ virus vaccine [2] and other vaccinations [3,4].

Furthermore, these patients suffer from high morbidity and mortality due to severe acute respiratory syndrome coronavirus 2 (SARS-CoV-2) $[5,6]$ and are at high risk of contracting the infection due to continuous visits to medical facilities and exposure to other people, even during peak times of the COVID-19 pandemic [7]. This study was undertaken to demonstrate the distinct humoral immune response in patients with kidney failure receiving maintenance hemodialysis. The antibody titers against the SARSCoV-2 spike (S) protein were evaluated in maintenance hemodialysis patients and compared with those in healthcare workers in the same center as controls.

\section{Methods}

\section{Study design}

\section{Study participants}

This study was conducted in our hemodialysis center with two cohorts: 1) patients receiving maintenance hemodialy- sis and 2) healthcare workers from the same dialysis center as controls. Participants received a dose of Covishield ChAdOxl nCoV-19 coronavirus vaccine (recombinant) developed by AstraZeneca (Cambridge, United Kingdom), and manufactured by Serum Institute of India Pvt Ltd. (Pune, India) from COVID-19 vaccination centers deputed by the government. All patients received the standard dose $(0.5$ $\mathrm{mL}$ ) of the vaccine containing $5 \times 10^{10}$ viral particles [8] A total of 141 patients was receiving maintenance hemodialysis in our center. At the time of the study, 20 patients had been vaccinated for approximately 6 to 7 weeks and were waiting for their scheduled second dose at 12 to 16 weeks, and 72 patients had been vaccinated 4 weeks prior. Twenty-one patients had a history of COVID-19 infection, 22 were not vaccinated at the time of the study, and six refused to get vaccinated. Participant selection is shown in Fig. 1.

Seventy-two patients included in the study were receiving maintenance hemodialysis in our center and had been vaccinated with the first dose 4 weeks prior. Subjects who were younger than 18 years, had a history of COVID-19 infection, were vaccinated previously at 6 weeks or more, or had an acute illness at the time of the study were excluded. In addition, 72 healthcare workers from the same dialysis center were enrolled as controls for the study.

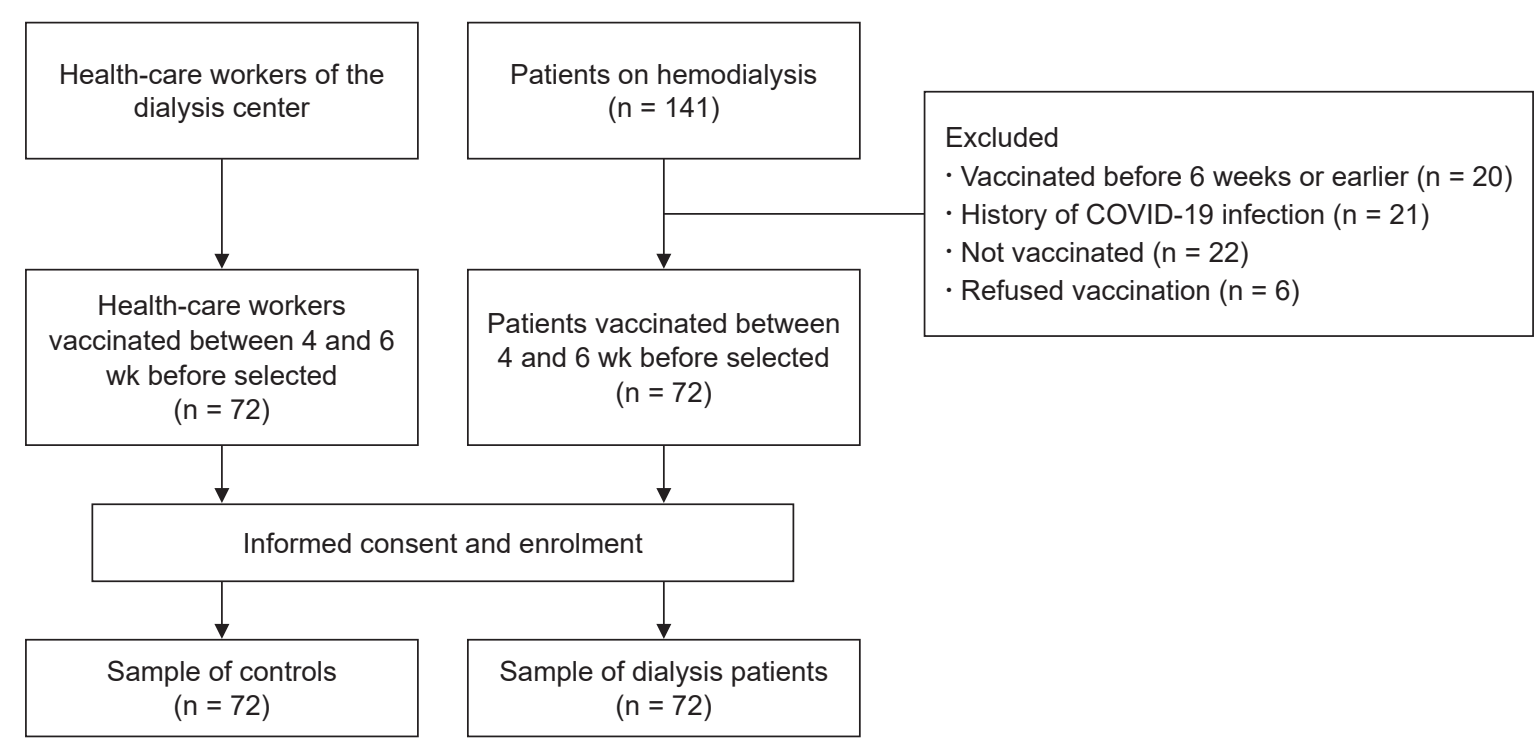

Figure 1. Study participant selection. All participants, including the controls, provided blood samples in the 5 th week after vaccination.

COVID-19, coronavirus disease 2019. 


\section{Antibody measurement}

The humoral immunological response was determined by measuring antibody titers using electrochemiluminescence immunoassay with Elecsys Anti-SARS-CoV-2 S on a Cobas 6000 analyzer e 601 module (Roche Diagnostics, Rotkreuz, Swiss) [9]. This test is an immunoassay for in vitro quantitative determination of antibodies (including immunoglobulin G [IgG]) to the SARS-CoV-2 S protein receptor-binding domain (RBD) in human serum and plasma. In previous studies, although full-length and fragments of S protein induced specific antibodies with neutralizing activity, the RBD was suggested as a major target for eliciting highly potent neutralizing antibodies with protective efficacy [10]. This test has a linear range of 0.4 to $250 \mathrm{U} / \mathrm{mL}$. Titers of $<0.8 \mathrm{U} / \mathrm{mL}$ were considered nonreactive and $\geq 0.8$ $\mathrm{U} / \mathrm{mL}$ reactive.

\section{Data and sample collection}

Informed consent was obtained from the participants including controls for drawing blood samples and participation in the study. Demographic details, medical history, and adverse events following vaccination of the patients and controls were collected with a proforma. Venous blood samples were taken from the patients during their dialysis visits before the initiation of the session.

Although the SARS-CoV-2 antibodies against S antigen and its subunits can be detected as early as 1 to 3 weeks after an infection or vaccination, the test used attains maximum sensitivity by the 5 th week [9]. Therefore, all samples were collected between 28 and 35 days after the first dose of the vaccine for both patients and controls. Body mass index (BMI) was defined as dry weight in kilograms divided by the square of the height in meters.

\section{Statistical analyses}

Statistical analysis was performed using the IBM SPSS ver. 23.0 (IBM Corp., Armonk, NY, USA). All categorical variables were presented as frequency and percentage and continuous variables as either mean (standard deviation) or median (interquartile range). The shape of the data distribution of antibody titers was assessed using the histogram/Shapiro-Wilk test. The chi-square test and Fisher exact test were used to determine the difference between the dialysis and control groups based on proportion values, and an independent sample $\mathrm{t}$ test or Mann-Whitney U test was used to determine the difference among the groups based on continuous values. Spearman correlation was used to determine the relationship between antibody titers and demographic and laboratory parameters. Multivariate logistic regression was used to determine the most important predictors of nonreactive antibody titers. BMI was classified based on Asian BMI classification guidelines. A $p$-value of $<0.05$ was considered statistically significant.

\section{Ethics statement}

The Institutional Ethics Committee in our hospital requires approval only for original research with trial/experimental interventions (procedure/drugs) administered patients (approval No. DHR Reg.No.EC/NEW/INST/2020/484). These patients were not treated with any trial/experimental therapy. All procedures followed the guidance of the Declaration of Helsinki. Written informed consent was obtained from the patients for participation in the study and for performing investigations.

\section{Results}

The dialysis and control groups each included 72 participants. The majority of patients in the dialysis group were males (80.6\%) and in the control group females (66.7\%). The average age in the dialysis group was higher $(46.14 \pm 8.89$ years) than in the control group ( $40.35 \pm 6.65$ years). Mean BMI was lower in the dialysis group $\left(20.87 \pm 4.26 \mathrm{~kg} / \mathrm{m}^{2}\right)$.

The majority of patients $(\mathrm{n}=48)$ had hypertension (66.7\%), 20 (27.8\%) had diabetes mellitus, four (5.6\%) had dilated cardiomyopathy, four (5.6\%) had a history of cerebrovascular accident, and two (2.8\%) had hypothyroidism. Among patients in the dialysis group, 36 (50.0\%) had hepatitis $\mathrm{C}$ virus (HCV) infection at the time of the study, and $26(36.1 \%)$ were receiving treatment. On average, the dialysis duration was $8.65 \pm 6.68$ months; four patients (5.6\%) had permanent catheters, and the remaining patients had arteriovenous fistula for hemodialysis access. None of the study participants were on or received immunosuppressive agents at least 3 months prior to the study.

Diabetic kidney disease was the most common native kidney disease, observed in 18 patients $(25.0 \%)$, followed by chronic glomerulonephritis in $14(19.4 \%)$, chronic in- 
terstitial nephritis in six (8.3\%), obstructive uropathy in five (6.9\%), hypertensive nephrosclerosis in three (4.2\%), and autosomal dominant polycystic kidney disease in two (2.8\%). Native kidney disease was not known in 24 (33.3\%) patients because many patients in India present with advanced stages of CKD. Among control subjects, four (5.6\%) had diabetes mellitus, and two (2.8\%) had hypertension. Demographic details, clinical characteristics, and laboratory results of the study subjects are shown in Table 1.

\section{Lower humoral response in the dialysis group}

All study subjects in the control group developed a positive humoral response defined based on a value of $\geq 0.8 \mathrm{U} / \mathrm{mL}$ compared with only 64 of $72(88.9 \%)$ in the dialysis group (Table 1). All eight patients with nonreactive antibody titers were older (greater than the mean age of the dialysis group), four were HCV infected, and two had diabetes mellitus.

\section{Adverse events}

Fever and myalgia were significantly more common in the control subjects (Table 2). Other adverse events such as pain at the local site, pruritus, or abdominal pain were observed in only a few patients. Serious adverse events were not observed in any study subjects.

\section{Factors affecting antibody titers}

In the dialysis group, age negatively correlated with antibody titer (Table 3); titer was lower with increasing age (Spearman correlation $\rho=-0.234$, $\mathrm{p}=0.04$ ). Dialysis dose $(\mathrm{Kt} / \mathrm{V})$ did not correlate with antibody titer $(\rho=0.085, \mathrm{p}=$ $0.75)$. Similarly, hemoglobin $(\rho=0.003, p=0.98)$ and albumin levels ( $\rho=0.147, p=0.22)$ did not correlate with titer. Bivariate analysis of laboratory results, sex, age, and BMI with antibody titers is shown in Table 3.

In the dialysis group, age and sodium level correlated with antibody titer in bivariate analysis; titers were lower in patients with higher age (Spearman correlation $\rho=-0.234$, $p=0.048)$ and lower sodium level $(\rho=0.237, p=0.045)$. Statistically significant correlation was not observed between antibody titers and other lab parameters.

Fisher exact test ( $p>0.05$ ) showed no significant difference in the proportion of reactive titer group subjects and nonreactive titer group subjects regarding HCV infection, diabetes mellitus, BMI, dialysis duration, lymphocyte count, and adverse events such as fever and myalgia (Table 4).

A multivariate logistic regression model was used to construct the statistical model to predict the risk of nonreactive antibody titers using the most significant two factors correlating with low antibody titers identified based on bivariate analysis, age, and sodium levels (Table 5). Only age (p $=0.045$ ) was a statistically significant independent variable associated with nonreactive antibody titers.

\section{Discussion}

The compromised immunity in hemodialysis patients is evident by a poor immune response to vaccinations such as hepatitis B [1], pneumococcus [2], or influenza [3]. In the present study, the humoral immune response to COVID-19 vaccination (Covishield) in hemodialysis patients compared with a cohort of healthcare workers in the same center was investigated.

Covishield is a recombinant, monovalent vaccine composed of a replication-deficient chimpanzee adenovirus (ChAdOx1) vector that encodes the $\mathrm{S}$ glycoprotein of SARS$\mathrm{CoV}-2$. The antigen is expressed locally, stimulating neutralizing antibodies and cellular immune responses. The initial schedule of Covishield ChAdOx1 nCoV-19 coronavirus vaccine (recombinant) was two doses of $0.5 \mathrm{~mL}$ each, 4 weeks apart, which was later spaced to 12 to 16 weeks for better coverage of a larger population and due to improved efficacy in clinical trials with increased spacing. Due to a short supply of vaccines, a policy of initially vaccinating a larger cohort with a single dose might provide better overall population protection than vaccinating half the number of individuals with two doses in the short term [1].

In our study, the antibody titers were significantly lower in the dialysis group, including eight patients (11.1\%) with absent antibody titer $(<0.8 \mathrm{U} / \mathrm{mL})$. This weaker antibody response was comparable with results obtained in studies with messenger RNA vaccines [11,12], in which approximately $10 \%$ of dialysis patients did not have an antibody response. Simon et al. [13] studied vaccine response in 86 dialysis patients and found 17 (19.8\%) were nonresponders even after the second dose, which was almost double than reported in our study. The study by Simon et al. [13] excluded patients who tested positive for antibodies before 
Table 1. Demographic details, clinical characteristics, and laboratory parameters of the study subjects

\begin{tabular}{|c|c|c|}
\hline Factor & Dialysis group & Control group \\
\hline \multicolumn{3}{|l|}{ Demographics } \\
\hline No. of patients & 72 & 72 \\
\hline \multicolumn{3}{|l|}{ Sex } \\
\hline Male & $58(80.6)$ & $24(33.3)$ \\
\hline Female & $14(19.4)$ & $48(66.7)$ \\
\hline Age (yr) & $46.14 \pm 8.89$ & $40.35 \pm 6.65$ \\
\hline \multicolumn{3}{|l|}{ Clinical characteristics } \\
\hline Body mass index $\left(\mathrm{kg} / \mathrm{m}^{2}\right)$ & $20.87 \pm 4.26$ & $23.12 \pm 3.35$ \\
\hline Diabetes mellitus & $20(27.8)$ & $4(5.6)$ \\
\hline Hypertension & $48(66.7)$ & $2(2.8)$ \\
\hline Dilated cardiomyopathy & $4(5.6)$ & - \\
\hline Cerebrovascular accident & $4(5.6)$ & - \\
\hline Hypothyroidism & $2(2.8)$ & - \\
\hline HCV infection & $36(50.0)$ & - \\
\hline \multicolumn{3}{|l|}{ Blood pressure (mmHg) } \\
\hline Systolic blood pressure & $145.81 \pm 15.10$ & $127.56 \pm 6.20$ \\
\hline Diastolic blood pressure & $94.77 \pm 10.63$ & $79.12 \pm 4.21$ \\
\hline \multicolumn{3}{|l|}{ Native kidney disease } \\
\hline Diabetic kidney disease & $18(25.0)$ & - \\
\hline Chronic glomerulonephritis & $14(19.4)$ & - \\
\hline Chronic interstitial nephritis & $6(8.3)$ & - \\
\hline Obstructive uropathy & $5(6.9)$ & - \\
\hline Hypertensive nephrosclerosis & $3(4.2)$ & - \\
\hline ADPKD & $2(2.8)$ & - \\
\hline Unknown & $24(33.3)$ & - \\
\hline \multicolumn{3}{|l|}{ Dialysis access } \\
\hline Arteriovenous fistula & $68(94.4)$ & - \\
\hline Permanent catheter & $4(5.6)$ & - \\
\hline Dialysis adequacy (Kt/V) & $1.16 \pm 0.17$ & - \\
\hline Dialysis duration (mo) & $8.65 \pm 6.68$ & - \\
\hline \multicolumn{3}{|l|}{ Laboratory parameters } \\
\hline Hemoglobin (g/dL) & $8.07 \pm 1.33$ & $12.69 \pm 1.27$ \\
\hline White blood cell count (cell/mm³) & $6,944.4 \pm 2,770.4$ & $8,204.2 \pm 1,991.0$ \\
\hline Neutrophils (\%) & $67.85 \pm 10.54$ & $58.11 \pm 9.13$ \\
\hline Lymphocytes (\%) & $22.49 \pm 14.16$ & $31.49 \pm 6.72$ \\
\hline Neutrophil-to-lymphocyte ratio & $3(2.09-5.26)$ & $1.79(1.38-2.57)$ \\
\hline Creatinine (mg/dL) & $7.15 \pm 3.29$ & $0.88 \pm 0.17$ \\
\hline Sodium (mEq/L) & $133.5 \pm 5.2$ & $136.5 \pm 4.0$ \\
\hline Potassium (mEq/L) & $4.49 \pm 1.05$ & $4.27 \pm 0.36$ \\
\hline Albumin (g/dL) & $3.03 \pm 0.70$ & $4.05 \pm 0.37$ \\
\hline \multicolumn{3}{|l|}{ Antibody titer (U/mL) } \\
\hline Reactive titer, $\geq 0.8$ & $64(88.9)$ & $72(100)$ \\
\hline Nonreactive titer, $<0.8$ & $8(11.1)$ & $0(0)$ \\
\hline
\end{tabular}

Data are expressed as number only, number (\%), mean \pm standard deviation, or median (interquartile range). ADPKD, autosomal dominant polycystic kidney disease; $\mathrm{HCV}$, hepatitis $\mathrm{C}$ virus. 
Table 2. Adverse events due to vaccination in dialysis and control groups

\begin{tabular}{lcc}
\hline Adverse event & Dialysis group $(n=72)$ & Control group $(n=72)$ \\
\hline Fever & $12(16.7)$ & $32(44.4)$ \\
Myalgia & $6(8.3)$ & $32(44.4)$ \\
Pruritus & $2(2.8)$ & $0(0)$ \\
Pain at the local site & $4(5.6)$ & $0(0)$ \\
Abdominal pain & $0(0)$ & $2(2.8)$
\end{tabular}

Data are expressed as number (\%).

Table 3. Spearman rank correlation between pathological parameters, sex, age, BMI, and antibody titers

\begin{tabular}{|c|c|c|}
\hline \multirow{2}{*}{ Variable } & \multicolumn{2}{|c|}{ Dialysis group } \\
\hline & $\rho$ & $\mathrm{p}$-value \\
\hline Sex & -0.131 & 0.27 \\
\hline Age & -0.234 & 0.05 \\
\hline $\mathrm{BMI}$ & 0.023 & 0.85 \\
\hline Hemoglobin & 0.003 & 0.98 \\
\hline White blood cell count & 0.144 & 0.23 \\
\hline Neutrophils & 0.016 & 0.89 \\
\hline Lymphocytes & -0.117 & 0.33 \\
\hline NLR & 0.123 & 0.30 \\
\hline Creatinine & 0.057 & 0.64 \\
\hline Sodium & 0.237 & 0.05 \\
\hline Albumin & 0.147 & 0.22 \\
\hline Dialysis dose $(\mathrm{Kt} / \mathrm{V})$ & 0.085 & 0.75 \\
\hline
\end{tabular}

BMI, body mass index; NLR, neutrophil-to-lymphocyte ratio; $\rho$, point-biserial.

vaccination, thereby eliminating subjects who developed antibodies due to subclinical infections. In contrast, all participants in the control group developed a humoral response, with the majority (89.9\%) having titer of $\geq 250 \mathrm{U} /$ $\mathrm{mL}$. This comparison clearly demonstrated a weaker antibody response in dialysis patients, indicating this group was susceptible to higher morbidity and mortality following infection.

Overall, the age of dialysis subjects enrolled in the present study was relatively younger ( $46.14 \pm 8.89$ years) compared with that of CKD patients. Most of the older patients in our center were vaccinated much earlier than 4 weeks because only older individuals were eligible for the initial round of vaccinations in India, hence, were not eligible for the study. In addition, fewer older patients were receiving hemodialysis in the dialysis unit because many older endstage renal disease patients refuse dialysis, and even those scheduled for maintenance hemodialysis typically do not
Table 4. Comparison of antibody titer levels in HCV infection, diabetes mellitus, and BMI

\begin{tabular}{|c|c|c|c|}
\hline Variable & Responder & Nonresponder & p-value \\
\hline \multicolumn{4}{|l|}{ HCV infection } \\
\hline Positive & $32(50.0)$ & $4(50.0)$ & \multirow[t]{2}{*}{$>0.999$} \\
\hline Negative & $32(50.0)$ & $4(50.0)$ & \\
\hline \multicolumn{4}{|c|}{ Diabetes mellitus } \\
\hline Positive & $18(28.1)$ & $2(25.0)$ & \multirow[t]{2}{*}{$>0.999$} \\
\hline Negative & $46(71.9)$ & $6(75.0)$ & \\
\hline \multicolumn{4}{|l|}{$\mathrm{BMI}^{\mathrm{a}}$} \\
\hline Underweight & $22(16.2)$ & $2(25.0)$ & \multirow[t]{4}{*}{0.05} \\
\hline Normal & $57(41.9)$ & $6(75.0)$ & \\
\hline Overweight & $47(34.6)$ & $0(0)$ & \\
\hline Obese & $10(7.4)$ & $0(0)$ & \\
\hline \multicolumn{4}{|c|}{ Dialysis duration (mo) } \\
\hline$\leq 5$ & $34(53.1)$ & $4(50.0)$ & \multirow[t]{2}{*}{$>0.999$} \\
\hline$>5$ & 30 (46.9) & $4(50.0)$ & \\
\hline \multicolumn{4}{|l|}{ Lymphocytes (\%) } \\
\hline$\leq 27$ & $72(52.9)$ & $6(75.0)$ & \multirow[t]{2}{*}{0.29} \\
\hline$>27$ & $64(47.1)$ & $2(25.0)$ & \\
\hline \multicolumn{4}{|c|}{ Significant adverse event } \\
\hline \multicolumn{4}{|l|}{ Fever } \\
\hline Present & $44(32.4)$ & $0(0)$ & \multirow[t]{2}{*}{0.11} \\
\hline Absent & $92(67.6)$ & $8(100)$ & \\
\hline \multicolumn{4}{|l|}{ Myalgia } \\
\hline Present & $38(27.9)$ & $0(0)$ & \multirow[t]{2}{*}{0.11} \\
\hline Absent & $98(72.1)$ & $8(100)$ & \\
\hline
\end{tabular}

Data are expressed as number (\%).

$\mathrm{BMI}$, body mass index; $\mathrm{HCV}$, hepatitis $\mathrm{C}$ virus.

aWorld Health Organization's Asian BMI classification.

Table 5. Factors associated with nonreactive antibody titers among the dialysis patients based on multivariate logistic regression analysis

\begin{tabular}{lcc}
\hline Predictor & OR $(95 \% \mathrm{Cl})$ & $p$-value \\
\hline Age & $1.11(1.01-1.22)$ & 0.05 \\
Sodium & $0.76(0.71-1.08)$ & 0.06
\end{tabular}

Dependent variable is antibody titers ( 1 , nonreactive; 0 , reactive). $\mathrm{Cl}$, confidence interval; OR, odds ratio.

receive dialysis regularly and are lost to follow-up due to economic burden on the family.

Age, sex, and BMI were distributed unequally between the control and dialysis groups; the majority of subjects were female in the control group and male in the dialysis group. Furthermore, the patients in the control group were younger on average and had higher BMI than subjects in 
the dialysis group.

A multivariate analysis was performed to account these confounding factors. Among the variables, only age affected the antibody titers with statistical significance, which was in agreement with various studies [13-16] showing similar relatively poor antibody responses in older age groups. This reinforces the importance of the age factor in the humoral response.

Bivariate analysis showed sodium to correlate with low antibody titer $(\rho=-0.237, \mathrm{p}=0.04)$ but was not significant in multivariate analysis including age and sodium level as variables. The correlation in bivariate analysis indicated the probable presence of cofounders. Hemodilution due to volume overload could have caused pseudo-low antibody titers because samples were collected before the dialysis session. Most patients in the study were presumed to have achieved dry weight because only subjects receiving hemodialysis for more than 2 months were enrolled in the study. However, volume status was assessed based on clinical examination, and objective methods such as body composition monitors were not used. Furthermore, hyponatremia in dialysis patients can be due to factors other than fluid overload, such as malnutrition and inflammation [17,18]. A detailed assessment is needed of nutritional status and inflammatory markers not tested in the present study, which are study's limitations.

A statistically significant association was not observed between antibody levels and sex, BMI, dialysis duration, dialysis dose, hemoglobin, albumin, total count, lymphocyte count, urea, liver function tests, HCV infection, diabetes mellitus, and adverse events due to vaccines. Grupper et al. [15] demonstrated a correlation between lymphocyte count and the humoral response given their role in adaptive immunity, which was not observed here; however, the authors also did not find an association with BMI, dialysis duration, dialysis dose, or albumin level.

Although the seroconversion rate may be considered relatively good for dialysis patients, it is subpar when compared to normal subjects. These patients are exposed repeatedly to other patients and healthcare workers due to their regular visits for dialysis and have high morbidity and mortality due to comorbidities.

Baseline antibody titers were not measured in the study participants. Although previously infected patients were excluded from our study, seroconversion due to subclinical infections or minimal symptoms might not have been evident. However, this confounding factor was at least partly addressed by selecting control subjects from the same hemodialysis center.

Neutralization assays such as the plaque reduction neutralization test (PRNT) are the gold standard for serological testing and determining immune protection against COVID-19 [19]. PRNT has several limitations rendering it unsuitable for large-scale studies. The assay used in the present study, Elecsys Anti-SARS-CoV-2 S, is not a neutralization test but has a good correlation with pseudovirus neutralization assay (safer and more versatile than neutralization assay), with a positive agreement of $92.3 \%$ (95\% confidence interval [CI], 63.97-99.81) [20]. This assay shows a positive predictive agreement of $96.6 \%$ (95\% CI, 93.35-98.51) and a negative predictive agreement of $99.98 \%$ (95\% CI, 99.91-100) [21].

SARS-CoV-2 antibodies against $S$ protein and its subunits can be detected within 1 to 3 weeks after infection $[22,23]$. However, we tested in the 5 th week after vaccination because our test attains maximum sensitivity by that period [9].

The upper limit of detection of Elecsys Anti-SARS-CoV-2 $\mathrm{S}$ assay is $250 \mathrm{U} / \mathrm{mL}$, a limitation of our study. A higher upper limit of the assay would be preferable because a better difference in adaptive humoral response between dialysis and the control groups could have been identified. Similarly, the complete humoral response profile after the second dose of the COVID-19 vaccine will provide relevant information but will require a larger sample and control cohorts. This was a single-center study with a limited number of patients, and complete humoral response was not the focus; however, differences were apparent.

Lack of follow-up of the patient titers is another limitation of our study. Even in normal subjects with adequate antibody responses, titers have been shown to decline over time [24]. In some studies, SARS-CoV-2 IgG titers decline substantially in dialysis patients within 3 months after diagnosis [25]. Therefore, these patients should be followed up with titers to monitor the level of immunity, which can help determine the need and timing of booster doses.

In addition to antibody production in response to SARSCoV-2 infection, host cellular immunity plays an essential role in impeding virus replication and expansion at various stages of COVID-19 disease [26]. CD4 and CD8 T 
cells recognize multiple regions of the $\mathrm{N}$ protein of SARS$\mathrm{CoV}-2$ in previously exposed patients. $\mathrm{T}$ cells showed strong cross-reactivity to the N protein of SARS-CoV-2 in patients who recovered from SARS (the disease associated with SARS-CoV infection) 17 years after the outbreak in 2003 [27]. Furthermore, patients who had no detectable antibody at 6 months after COVID-19 infection were found to have SARS-CoV-2 antigen-specific T-cell responses [28]. Our study did not attempt to determine the T-cell immune response, which might contribute to protection from COVID-19.

In conclusion, our study results demonstrated a weak antibody response of hemodialysis patients to the viral vector COVID-19 vaccine, and older age was associated with nonresponders. Evaluation of both humoral and cellular immunity after the second dose of COVID-19 vaccine and serial follow-up of antibody titers can help determine the need for booster shots.

\section{Conflicts of interest}

All authors have no conflicts of interest to declare.

\section{Authors' contributions}

Conceptualization: AR, NR

Data curation: NR, RD

Formal analysis: $\mathrm{MR}, \mathrm{RD}$

Investigation: $\mathrm{NR}, \mathrm{AP}$

Methodology: AR, NR

Project administration: NR, JA

Visualization: NR

Writing-original draft: NR

Writing-review \& editing: all authors

All authors read and approved the final manuscript.

\section{ORCID}

Niranjan Raja, https://orcid.org/0000-0001-6866-4098

Arul Rajagopalan, https://orcid.org/0000-0002-3403-0780

Jegan Arunachalam, https://orcid.org/0000-0002-7420-0123

Arun Prasath, https://orcid.org/0000-0002-4937-835X

Rakesh Durai, https://orcid.org/0000-0002-7276-3440

Manorajan Rajendran, https://orcid.org/0000-0002-4032-0186

\section{References}

1. Voysey M, Costa Clemens SA, Madhi SA, et al. Single-dose administration and the influence of the timing of the booster dose on immunogenicity and efficacy of ChAdOx1 nCoV-19 (AZD1222) vaccine: a pooled analysis of four randomised trials. Lancet 2021;397:881-891.

2. Stevens CE, Alter HJ, Taylor PE, Zang EA, Harley EJ, Szmuness W. Hepatitis B vaccine in patients receiving hemodialysis. Immunogenicity and efficacy. N EnglJ Med 1984;311:496-501.

3. Fuchshuber A, Kühnemund O, Keuth B, Lütticken R, Michalk D, Querfeld U. Pneumococcal vaccine in children and young adults with chronic renal disease. Nephrol Dial Transplant 1996;11:468-473.

4. Scharpé J, Peetermans WE, Vanwalleghem J, et al. Immunogenicity of a standard trivalent influenza vaccine in patients on long-term hemodialysis: an open-label trial. Am J Kidney Dis 2009;54:77-85.

5. Goicoechea M, Sánchez Cámara LA, Macías N, et al. COVID-19: clinical course and outcomes of 36 hemodialysis patients in Spain. Kidney Int 2020;98:27-34.

6. Smolander J, Bruchfeld A. The COVID-19 epidemic: management and outcomes of hemodialysis and peritoneal dialysis patients in Stockholm, Sweden. Kidney Blood Press Res 2021;46:250-256.

7. Hsu CM, Weiner DE. COVID-19 in dialysis patients: outlasting and outsmarting a pandemic. Kidney Int 2020;98:1402-1404.

8. Voysey M, Clemens SA, Madhi SA, et al. Safety and efficacy of the ChAdOx1 nCoV-19 vaccine (AZD1222) against SARS-CoV-2: an interim analysis of four randomised controlled trials in Brazil, South Africa, and the UK. Lancet 2021;397:99-111.

9. Roche Diagnostics International Ltd. Elecsys Anti-SARS-CoV-2 S factsheet [Internet]. Rotkreuz (Switzerland): Roche Diagnostics International Ltd.; c2021 [cited 2021 Aug 19]. Available from: https://diagnostics.roche.com/content/dam/diagnostics/ Blueprint/en/pdf/cps/Elecsys-Anti-SARS-CoV-2-S-factsheetSEPT-2020-2.pdf.

10. Wang N, Shang J, Jiang S, Du L. Subunit vaccines against emerging pathogenic human coronaviruses. Front Microbiol 2020;11:298.

11. Lacson E Jr, Argyropoulos CP, Manley HJ, et al. Immunogenicity of SARS-CoV-2 vaccine in dialysis. J Am Soc Nephrol 2021;32:2735-2742.

12. Yanay NB, Freiman S, Shapira M, et al. Experience with SARSCoV-2 BNT162b2 mRNA vaccine in dialysis patients. Kidney Int 
2021;99:1496-1498.

13. Simon B, Rubey H, Treipl A, et al. Haemodialysis patients show a highly diminished antibody response after COVID-19 mRNA vaccination compared with healthy controls. Nephrol Dial Transplant 2021;36:1709-1716.

14. Agur T, Ben-Dor N, Goldman S, et al. Antibody response to mRNA SARS-CoV-2 vaccine among dialysis patients: a prospectivecohort study. Nephrol Dial Transplant 2021 Apr 11 [Epub]. DOI: 10.1093/ndt/gfab155.

15. Grupper A, Sharon N, Finn T, et al. Humoral response to the Pfizer BNT162b2 vaccine in patients undergoing maintenance hemodialysis. Clin J Am Soc Nephrol 2021;16:1037-1042.

16. Frantzen L, Cavaillé G, Thibeaut S, El-Haik Y. Efficacy of the BNT162b2 mRNA COVID-19 vaccine in a haemodialysis cohort. Nephrol Dial Transplant 2021;36:1756-1757.

17. Dekker MJ, Marcelli D, Canaud B, et al. Unraveling the relationship between mortality, hyponatremia, inflammation and malnutrition in hemodialysis patients: results from the international MONDO initiative. Eur J Clin Nutr 2016;70:779- 784.

18. Rhee CM, Ayus JC, Kalantar-Zadeh K. Hyponatremia in the dialysis population. Kidney Int Rep 2019;4:769-780.

19. Bewley KR, Coombes NS, Gagnon L, et al. Quantification of SARS-CoV-2 neutralizing antibody by wild-type plaque reduction neutralization, microneutralization and pseudotyped virus neutralization assays. Nat Protoc 2021;16:3114-3140.

20. Meyer B, Torriani G, Yerly S, et al. Validation of a commercially available SARS-CoV-2 serological immunoassay. Clin Microbiol
Infect 2020;26:1386-1394.

21. Roche Diagnostics. Elecsys Anti-SARS-CoV-2 S (Package Insert 2021-05, V2.0; Material Numbers 09289267190 and 09289275190) [Internet]. Mannheim (Germany): Roche Diagnostics; 2021 [cited cited 2021 Aug 19]. Available from: https:// www.fda.gov/media/144037/download.

22. Qu J, Wu C, Li X, et al. Profile of immunoglobulin G and IgM antibodies against severe acute respiratory syndrome coronavirus 2 (SARS-CoV-2). Clin Infect Dis 2020;71:2255-2258.

23. Wölfel R, Corman VM, Guggemos W, et al. Virological assessment of hospitalized patients with COVID-2019. Nature 2020;581:465-469.

24. Long QX, Tang XJ, Shi QL, et al. Clinical and immunological assessment of asymptomatic SARS-CoV-2 infections. Nat Med 2020;26:1200-1204.

25. Seow J, Graham C, Merrick B, et al. Longitudinal observation and decline of neutralizing antibody responses in the three months following SARS-CoV-2 infection in humans. Nat Microbiol 2020;5:1598-1607.

26. Wang X, Gui J. Cell-mediated immunity to SARS-CoV-2. Pediatr Investig 2020;4:281-291.

27. Le Bert N, Tan AT, Kunasegaran K, et al. SARS-CoV-2-specific T cell immunity in cases of COVID-19 and SARS, and uninfected controls. Nature 2020;584:457-462.

28. Clarke CL, Prendecki M, Dhutia A, et al. Longevity of SARS$\mathrm{CoV}-2$ immune responses in hemodialysis patients and protection against reinfection. Kidney Int 2021;99:1470-1477. 